\title{
MODELLING PEDESTRIAN CROSSING BEHAVIOUR AT A MIDBLOCK SECTION
}

\author{
Yugendar POOJARI ${ }^{1}$, Prashanth E. ${ }^{2}$, D. DIVYA ${ }^{2}$, Kalyani M. ${ }^{2}$
}

\begin{abstract}
In general, pedestrians want to cross a road at a mid-block section instead of intersections because it is the shortest route. According to an Indian scenario, most of the crosswalks in urban areas are not signalised and controlled. In this study, the crossing behaviour of pedestrians in terms of speed at a midblock section was studied by considering several parameters. Statistical tests such as ANOVA and Pearson correlation tests were performed in order to know the effect of parameters on the crossing speed of a pedestrian. The pedestrian's age and gender, crossing type, pattern, time, number of stops, number of two wheelers, three wheelers, light and heavy commercial vehicles, and groups significantly affect their crossing speed. A multiple linear regression model was developed by considering the most significant parameters. The mean absolute percentage error, root mean square error, and $R 2$ values were calculated to validate the models.
\end{abstract}

Address

1 Dept. of Civil Engineering, Chaitanya Bharathi Institute of Technology, Telangana, India

2 Dept. of Civil Engineering, Chaitanya Bharathi Institute of Technology, Telangana, India

* Corresponding author: pyugendar_civil@cbit.ac.in

Key words

- Age;

- Gender;

- Group; Luggage; Speed, ANOVA.

\section{INTRODUCTION}

Pedestrian safety must be the first priority in a transportation planning group. In developing countries, pedestrian safety is a major problem in urban areas due to speedy urbanization and increases in the vehicular population. A lot of factors influence pedestrians while they are crossing a road. A pedestrian crossing a road makes decisions as to whether to accept certain gaps between the flow of approaching vehicles. Pedestrian actions continuously change with respect to environmental characteristics, and their vulnerability is enhanced with increases in motorized traffic. The jaywalking behavior of a pedestrian is greater when crossing at a mid-block section compared to walking on sidewalks. A higher number of conflicts occur at mid-block locations due to jaywalking, which leads to more pedestrian fatalities. In developing countries such as India, pedestrians need to cross median openings due to the absence of footbridges and subways. Generally, pedestrians judge safe gaps in a traffic stream when crossing a road at midblock sections. The pedestrian crossing process at an uncontrolled intersection involves two aspects: first the pedestrian approaches the crossing facility, and second, performs the crossing maneuver. The crossing maneuver process involves the pedestrian departing from one curb to another curb across the roadway. Generally, pedestrians wait at curbs before starting to cross the road to view the available gaps. Then pedestrians will decide whether to accept or reject any gaps for crossing safely based on previous experience.

In general, a pedestrian's speed is $1.2 \mathrm{~m} / \mathrm{s}$ without any restrictions. According to the Traffic Engineering Handbook (Dewar 1992), a speed of $0.91-0: 98 \mathrm{~m} / \mathrm{s}$ is recommended for the design of pedestrian facilities. The Institute of Transportation Engineers (ITE Committee 4A-6 1983) suggests a speed of $0.75 \mathrm{~m} / \mathrm{s}$ when there is a greater number of older pedestrians at a location. The Highway Capacity Manual [Transportation Research Board (TRB) 2000] suggests $1.2 \mathrm{~m} / \mathrm{s}$ if the older pedestrian population is less than $20 \%$; otherwise, $1.0 \mathrm{~m} / \mathrm{s}$ is advisable. The Manual of Uniform Traffic Control Devices for Streets and Highways (MUTCD) [Federal Highway Administration (FHWA) 2003] suggests $1.21 \mathrm{~m} / \mathrm{s}$ from the curb to the other side of a roadway. The 
crossing speeds suggested by different organizations are different and vary with a range of $0.75-1.21 \mathrm{~m} / \mathrm{s}$. At crosswalk locations, pedestrians adjust their crossing speed due to accepting critical gaps and also the speed and type of the approaching vehicle. The quality of the pedestrian facilities in developing countries is very low compared to developed countries due to the limited availability of funding. Because of this, pedestrians related accidents are increasing at midblock sections due to heterogeneous traffic conditions (Leather, et al. 2011). According to (Mohan, et al. 2009), $60 \%$ of pedestrian fatalities occur in urban areas, and out of these, $85 \%$ occur at mid-block sections. The walking speed of pedestrians also varies with the environmental conditions and vehicular traffic flow (Chandra and Bharti, 2013). The crossing location is important and leads to risks for pedestrians during complex pedestrian crossing processes. In this case, traffic related factors (volume, speed, etc., of a vehicle), associated with physical space (number of lanes, road width, etc.), the interactions between pedestrians, and between pedestrians and vehicles as well as the behavior and psychological state of drivers and pedestrians have significant roles (Chu, et al. 2004; Papadimitriou, et al. 2009; Rajat, et al. 2011; Zhuang and Wu, 2011; Kadali and Vedagiri, 2013b; Yannis, Papadimitriou \& Theofilatos, 2013; Shaaban, Muley \& Mohammed, 2018; Zhao, Malenje, Tang \& Han, 2019).

Pedestrian walking speed also depends on demographic parameters such as age, gender and pedestrians with and without luggage (Fruin 1981, Tanaboriboon 1987, Holland and Hill 2007, Laxman, et al. 2010, Chandra, et al. 2014). Holland and Hill (2007) observed that male pedestrians perceived less risk compared to female pedestrians. Lam and Cheung (2000) observed in Hong Kong that pedestrian crossing speeds at crosswalks with a midblock section were less than crosswalks without a midblock section. Daamen and Hoogendorn (2007) observed that the crossing speeds of older female pedestrians are the slowest, i.e., 1.18 $\mathrm{m} / \mathrm{s}$. Tarawneh (2001) observed that female pedestrian crossing speeds $(1.33 \mathrm{~m} / \mathrm{s})$ were less than male pedestrian speeds $(1.35 \mathrm{~m} / \mathrm{s})$ in Jordan. A study carried out by IIT Delhi entitled "Road safety in India: challenges and opportunities" states that $58 \%$ of victims in urban areas in Mumbai and $47 \%$ in Delhi relate to pedestrian fatalities that occur on a road (Mohan et al., 2015). The Ministry of Road Transport and Highways (MoRTH) and World Health Organisation (WHO) estimate that the percentage of pedestrian and bicycle fatalities constitute $12 \%-13 \%$ of the total road traffic injuries (RTI) and fatalities in the country. Based on the above, it is clear that pedestrian safety is a main issue for transport planners, traffic engineers, and policymakers. It is therefore worth studying the road crossing behavior of pedestrians in mixed traffic conditions at uncontrolled mid-block locations. This study can help with understanding the pedestrian crossing behaviour on a micro- scopic level and also add to the safety of the vehicular traffic and pedestrians.

\section{STUDY AREA AND DATA COLLECTION}

A field survey was carried out at Langer House, Hyderabad, India, to study the crossing behaviour of pedestrians. This location was observed on different days to ascertain the peak volume of pedestrians and vehicles. It is a place with many hostels, which provide temporary shelters for college students, and with many commercial shops on either side of the road. This feature forces people to cross the road through the midblock. A high-rise building was selected adjacent to the midblock, and the survey was carried out by placing a camera on a supporting stand on top of the three-storey building. The aim of the survey was to videotape those pedestrians who intended to cross through the midblock. The data was collected continuously for 3 days from 7AM to 10AM. The data collected include individual characteristics such as the gender and age of the pedestrians, the number of crossing attempts, the type of crossing pattern followed, and the size of the group. The speed of incoming vehicles was measured by conducting a spot speed study. The width of the road was measured for calculating the crossing speeds. Figure 1 shows the mid-block section study area.

\section{DATA EXTRACTION}

The video produced was imported into Avidemux software which is an open source software for calculating the crossing time of pedestrians at midblock sections. The data extraction was done manually, and 980 samples were collected from the data. Various parameters extracted from the video included the age and gender of the pedestrians, persons with and without luggage, crossing patterns, etc. The next section describes the analysis; in the subsequent section the development of the model is discussed.

\section{ANALYSIS}

The observations from the data collected are shown in Figure 2. It was observed that a greater number of younger people were crossing the road compared to other age groups. The figure shows that the number of female pedestrians crossing the midblock were greater compared to the male pedestrians. Pedestrians without luggage crossing the road were much more observed compared to pedestrians with luggage. The figure also shows that a greater
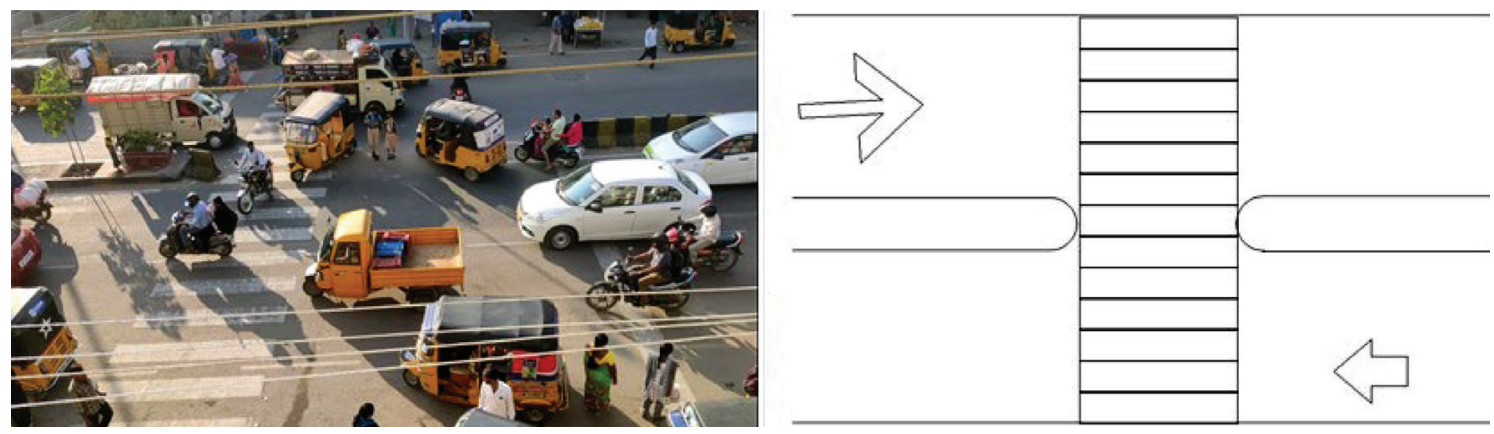

Fig. 1 Study Area 

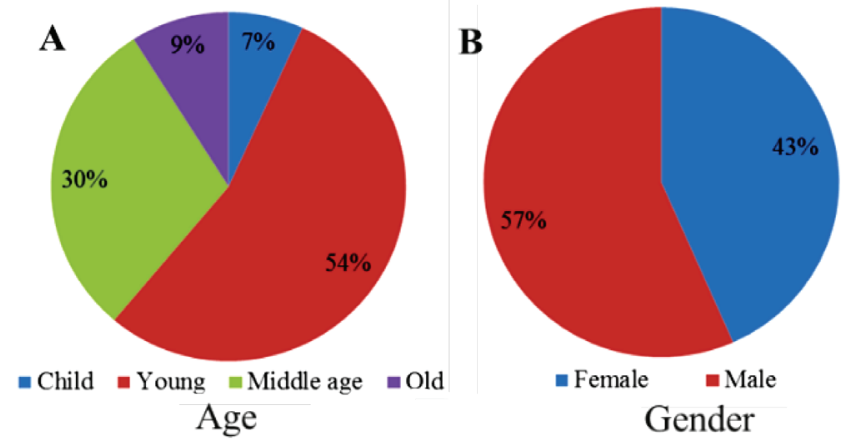

Gender

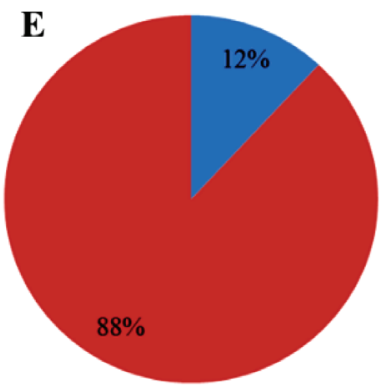

- Uninterrupted $\quad$ Interrupted Crossing Behaviour

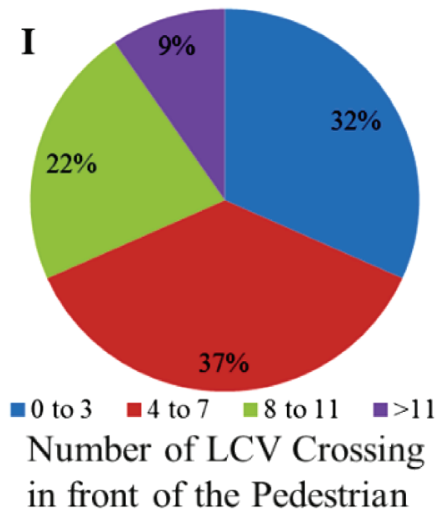

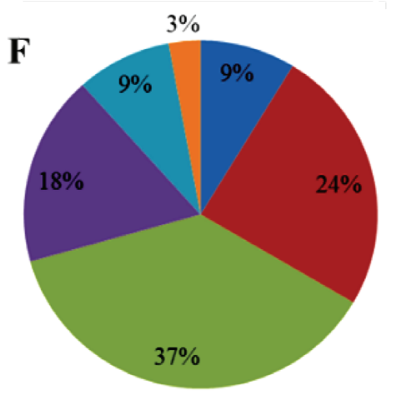

$0=1$
$m b e r$

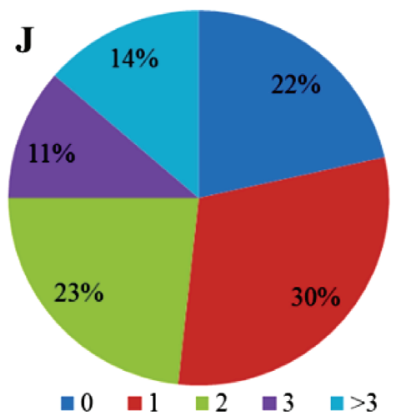

Number of HCV Crossing in front of the Pedestrian
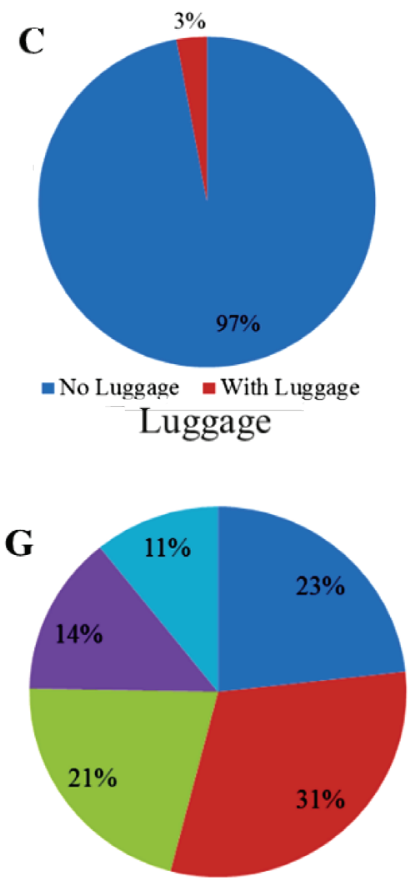

- 6 to $10=11$ to $15=16$ to $20=20$ Number of $2 \mathrm{~W}$ Crossin in front of the Pedestrian

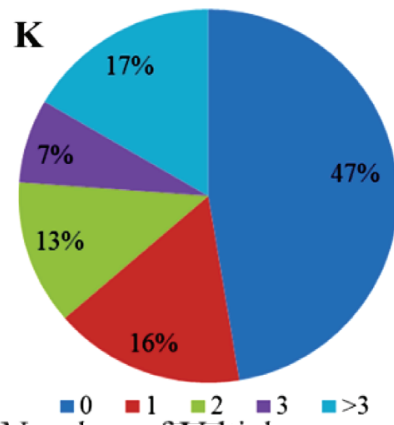

Number of Vehicles making U-turns

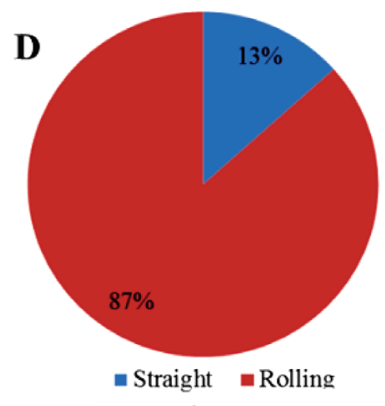

Crossing Pattern

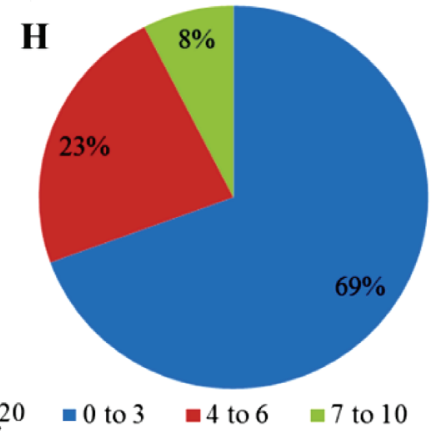

Number of $3 \mathrm{~W}$ Crossing in front of the Pedestriau L

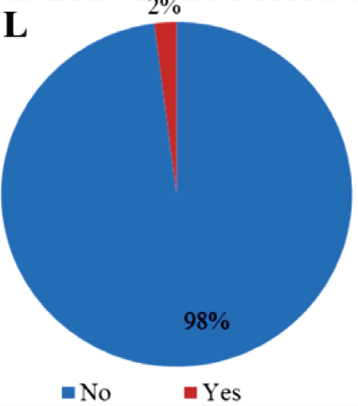

Mobile Usage

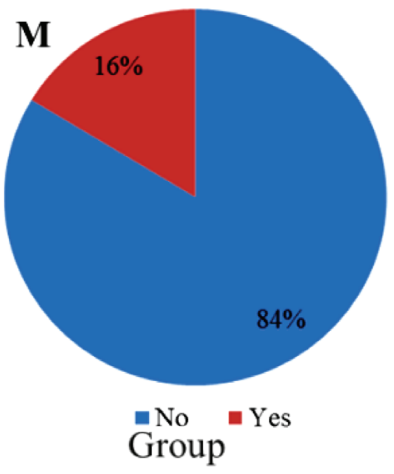

Fig. 2 Proportions of data observed

number of pedestrians were following a rolling pattern compared to a straight one. It was seen that a greater number of pedestrians were interrupted while crossing the road. From Figure 2 (F), it can be observed that more pedestrians stopped once while crossing the road compared to others. While pedestrians were crossing the road, it was observed that at least 6 to 10 two wheeler (2W) vehicles were crossing in front of the pedestrian (Figure $2(\mathrm{G})$ ). It was found that a greater number of three-wheeler $(3 \mathrm{~W})$ vehicles were crossing in front of the pedestrian while the pedestrians were crossing the road (Figure 2(H)). In the same way, it was observed that, a minimum of 4 to 7 light commercial vehicles (LCV) and a minimum of 1 heavy commercial vehicles (HCV) were crossing while the pedestrians were crossing the road (Figure 2(I \& J)). It can be seen that more than 3 vehicles took U-turns when pedestrians were crossing the road (Figure 2(K)). From Figure 2(L), it can be seen that a lower number of pedestrians were using a mobile phone while crossing the road. From Figure 2(M), it can be seen that more single pedestrians crossed the road compared to groups. 

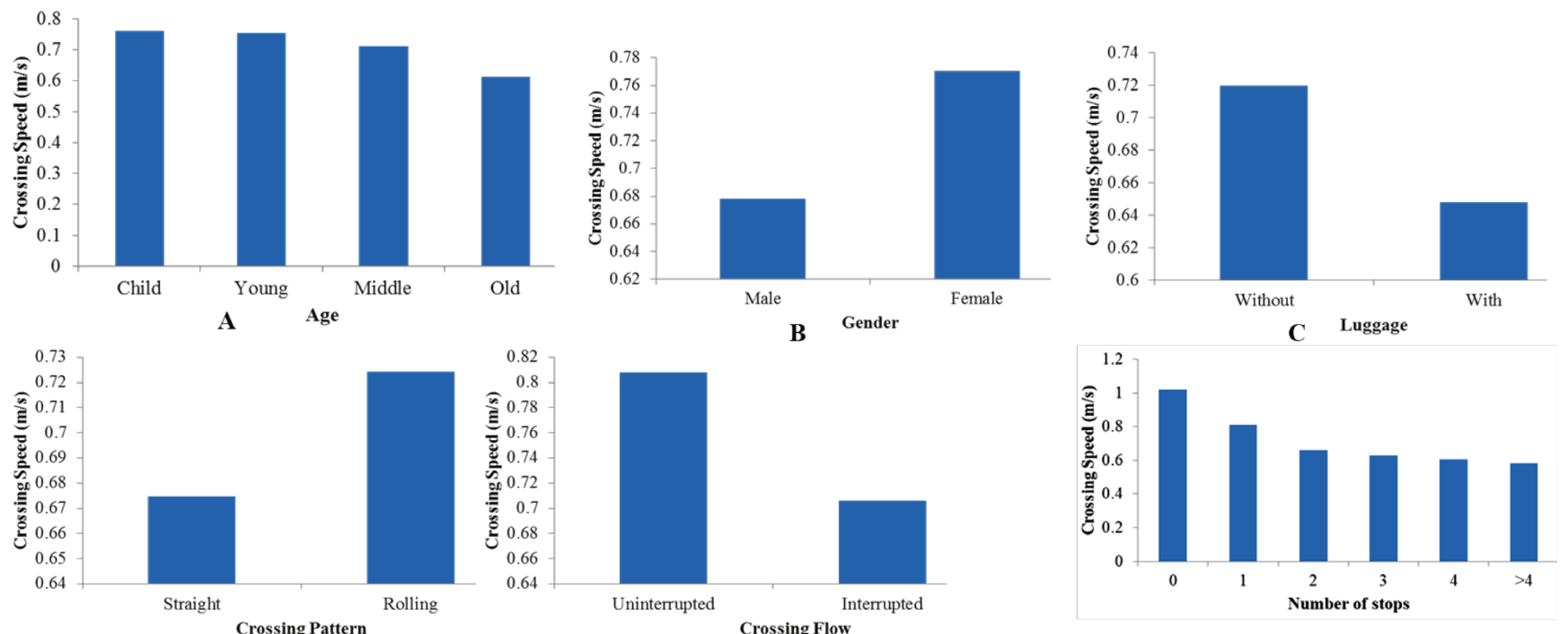

D
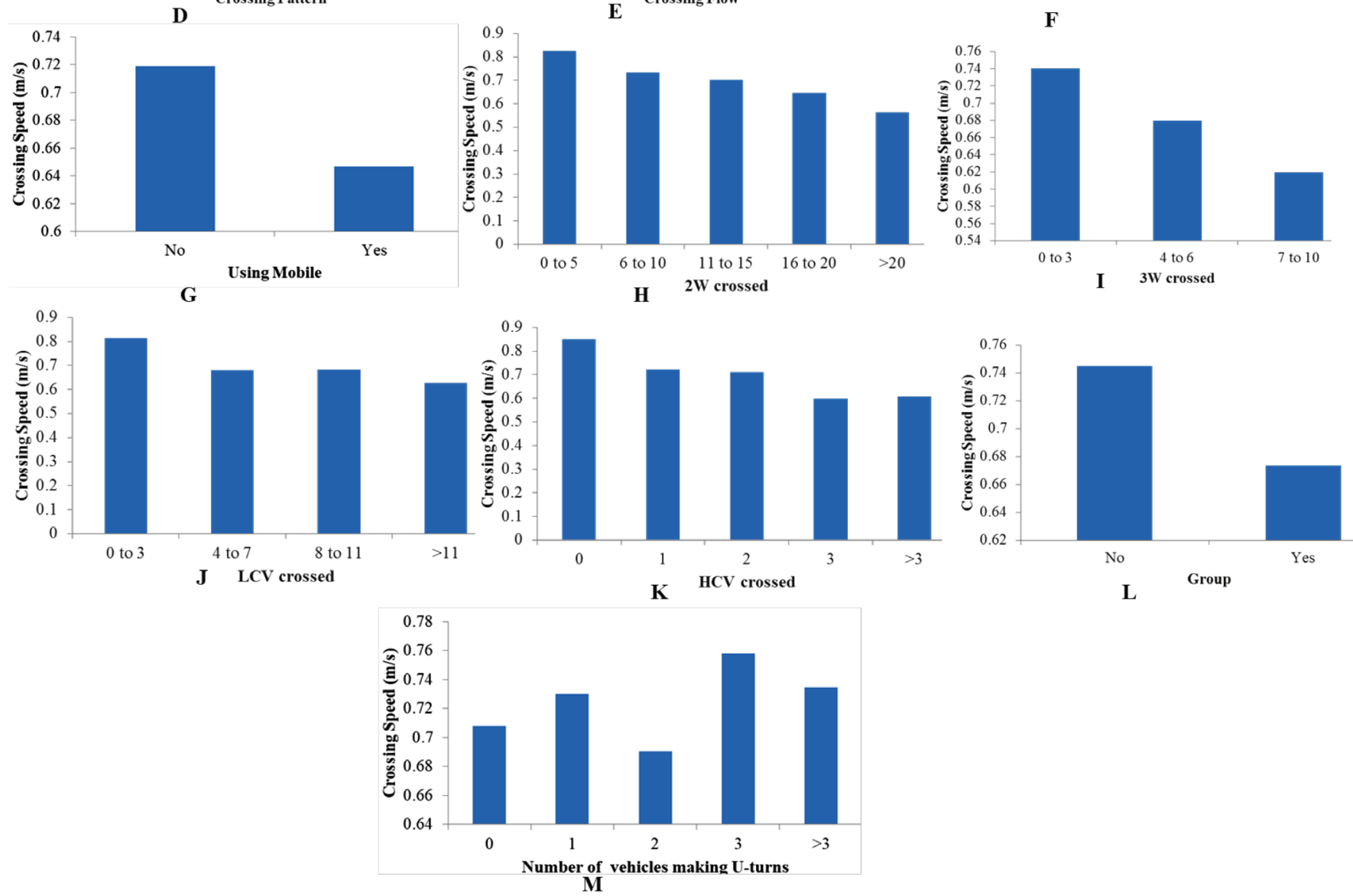

Fig. 3 Pedestrian average speed data for different parameters

From Figure 3(A), it can be seen that the crossing speed of older pedestrians was slower than other age groups. Figure 3(B) shows the crossing speed of male and female pedestrians. It shows female pedestrians were crossing at a higher speed than male pedestrians. Figure 3(C) shows the speed of pedestrians carrying and not carrying luggage. It can be seen that pedestrians without luggage cross faster than pedestrians with luggage. Figure 3(D) shows the crossing speed of pedestrians with different crossing patterns. Pedestrians who follow a rolling pattern crossing were faster than pedestrians who follow a straight pattern while crossing the road. Figure 3(E) shows the crossing speeds of pedestrians with interrupted or uninterrupted crossing behavior. It was observed that pedestrians who were uninterrupted crossed more quickly than pedestrians who were interrupted while crossing the road. Figure 3(F) shows the crossing speeds of pedestrians with interruptions and the number of times they stopped. It shows that pedestrian crossing speeds were greater for pedestrians who did not stop while crossing the road. Figure $3(\mathrm{G})$ shows the crossing speed of pedestrians using mobile phones. It was observed that the crossing speeds of pedestrians not using mobile phones were higher than pedestrians using mobiles. Figure $3(\mathrm{H})$ shows the crossing speed of pedestrians due to the two-wheeler vehicles moving on the road during the crossing time. It can be seen that pedestrian crossing speeds were higher if a fewer number of 
two-wheeler vehicles were moving on the road i.e, (0 to 5). Figure 3(I) shows the crossing speed of pedestrians with the number of three-wheeler vehicles moving on the road during the crossing time. It can be seen that the pedestrian crossing speeds were higher if a fewer number of three-wheeler vehicles moved on the road, i.e., (0 to 3). Figure 3(J) shows the crossing speed of pedestrians with the number of light commercial vehicles moving on the road during the crossing time. It shows that pedestrian crossing speeds were higher if a lower number of light commercial vehicles were moving on the road, i.e., (0 to 3). Figure 3(K) shows the crossing speed of pedestrians with the number of heavy commercial vehicles moving on the road during the crossing time. It can be seen that pedestrian crossing speeds were higher when no heavy commercial vehicles were moving on the road. Figure 3(L) shows the crossing speeds of pedestrians with the number of vehicles making u-turns. Pedestrian crossing speeds were greater in the absence of vehicles making u-turns. Figure 3(M) shows the crossing speed of pedestrians in and not in a group. It can be seen that a single pedestrian crosses faster than pedestrians in a group.

\section{STATISTICAL TESTS}

ANOVA and Pearson correlation tests were performed for statistical analyses, using SPSS. The variables considered for the analyses were the gender and age group of the pedestrians, the type of pattern while crossing, the type of crossing, i.e., interrupted or uninterrupted, the number of times pedestrians stopped while crossing the road, the effects of vehicles, the effects of mobiles, the effects of groups and pedestrians with or without luggage were factors. Gender was divided into two categories, and the values were assumed as 0 for a female pedestrian and 1 for a male pedestrian. Age was divided into four categories, and the values were considered as 0 for a child and 1 for younger, 2 for middle aged, and 3 for older pedestrians. The group size was divided into two categories, i.e., 0 for a single pedestrian and 1 for a group consisting of at least two members. Mobile phone usage was divided into two categories, i.e., 0 for not using a mobile and 1 for using a mobile. All the tests were performed at a $95 \%$ confidence level. From Table 1, it can be seen that a person's gender and age, group, rolling pattern, crossing type, and vehicle crossing factors have a significant effect on a pedestrians crossing speed. Table 1 shows the $\mathrm{F}$ value is greater than the table value for all the categories except luggage and mobiles and the $\mathrm{P}$ value is less than 0.05 for all the factors except using mobiles and luggage. The Pearson correlation coefficient value ranges between -1 and +1 , where -1 shows a totally negative correlation; 0 is a nonlinear correlation; and +1 shows a totally positive correlation. The Pearson correlation coefficients were found to be negative for factors such as gender and age, luggage, crossing type, number of stops, number of $2 \mathrm{~W}$ crossings, number of $3 \mathrm{~W}$ crossings, LCVs, HCVs, using a mobile, group size and crossing times. A nonlinear correlation was observed for the patterns, and the number of u-turning vehicles, see Table 2 . There was a significant effect on crossing speeds as the $\mathrm{p}$ value was found to be $<0.05$ for all the factors except luggage and using mobiles. Female pedestrian crossing speeds were greater compared to male pedestrian speeds. It was observed that older pedestrians have the slowest speed compared to the others. A person with a rolling pattern was faster than a person with a straight pattern. Pedestrians without groups were faster than persons with groups.

\section{MODELLING}

Eighty percentage of the data was used for developing the model, and the remaining $20 \%$ was used for validating the model.

The general form of the linear regression model suggested is

$$
\mathrm{B}=\mathrm{A}_{1} \mathrm{X}_{1}+\mathrm{A}_{2} \mathrm{X}_{2}+\ldots+\mathrm{A}_{\mathrm{k}} \mathrm{X}_{\mathrm{K}}+\varepsilon
$$

where $\mathrm{B}=$ speed, $\mathrm{X}_{1}=$ gender, $\mathrm{X}_{2}=$ age, $\mathrm{X}_{3}=$ crossing, $\mathrm{A}=$ coefficient, $\varepsilon=$ constant

Tab. 1 ANOVA statistical tests for pedestrian crossing movement

\begin{tabular}{|c|c|c|c|c|}
\hline \multirow[b]{2}{*}{ Factor } & \multicolumn{4}{|c|}{ ANOVA } \\
\hline & $\mathbf{F}$ & F critical & $\mathbf{P}$ & Remarks \\
\hline Age & 26.33 & 1.195 & 0.00 & Significant \\
\hline Gender & 12.02 & 1.195 & 0.00 & Significant \\
\hline Luggage & 0.498 & 0.837 & 0.34 & Not significant \\
\hline Pattern & 2.045 & 1.195 & 0.00 & Significant \\
\hline Crossing type & 2.145 & 1.195 & 0.00 & Significant \\
\hline Number of stops & 27.124 & 1.195 & 0.00 & Significant \\
\hline Number of $2 \mathrm{~W}$ crossed & 868.06 & 1.20 & 0.00 & Significant \\
\hline Number of $3 \mathrm{~W}$ crossed & 84.37 & 1.195 & 0.00 & Significant \\
\hline Number of LCVs crossed & 284.2292 & 1.195 & 0.00 & Significant \\
\hline Number of HCVs crossed & 43.95 & 1.195 & 0.00 & Significant \\
\hline Number of vehicles making U-turns & 59.33 & 1.195 & 0.00 & Significant \\
\hline Using a mobile & 0.351 & 0.837 & 0.31 & Not significant \\
\hline Group & 3.9 & 1.195 & 0.00 & Significant \\
\hline Crossing time & 1594.47 & 1.195 & 0.00 & Significant \\
\hline
\end{tabular}


Tab. 2 correlation statistical tests for movement of crossing pedestrian

\begin{tabular}{|l|l|l|l|}
\hline \multirow{2}{*}{ Factor } & \multicolumn{3}{|l|}{ Pearson correlation } \\
\cline { 2 - 4 } & Coefficient & P & Remarks \\
\hline Age & -0.158 & 0.00 & Significant \\
\hline Gender & 0.007 & 0.015 & Significant \\
\hline Luggage & -0.05 & 0.34 & Not significant \\
\hline Pattern & 0.07 & 0.67 & Not significant \\
\hline Crossing type & -0.15 & 0.00 & Significant \\
\hline Number of stops & -0.424 & 0.00 & Significant \\
\hline Number of 2W crossed & -0.365 & 0.00 & Significant \\
\hline Number of 3W crossed & -0.183 & 0.00 & Significant \\
\hline Number of LCVs crossed & -0.25 & 0.00 & Significant \\
\hline Number of HCVs crossed & -0.337 & 0.00 & Significant \\
\hline $\begin{array}{l}\text { Number of vehicles } \\
\text { making U-turns }\end{array}$ & 0.03 & 0.42 & Not significant \\
\hline Using a mobile & -0.043 & 0.31 & Not significant \\
\hline Group & -0.166 & 0.00 & Significant \\
\hline Crossing time & -0.886 & 0.00 & Significant \\
\hline
\end{tabular}

For the location studied, a regression analysis was applied to the pedestrian movement as tabulated in Table 3. Negative values of A were obtained that would significantly decrease the speed with every increase in the following parameters; children, females, persons in a group, crossing type, number of stops, number of $2 \mathrm{~W}$, number of LCV and number of HCV. The pedestrian crossing type has a greater effect on the speed of the people in a group compared to others because the coefficient of the crossing type is more compared to the other factors.

Tab. 3 Regression analysis for the pedestrian crossing speed

\begin{tabular}{|l|l|l|}
\hline Model & Coefficient (A) & Significance \\
\hline Constant & 1.4418 & 0.000 \\
\hline Age & 0.001 & 0.000 \\
\hline Gender & -0.021 & 0.000 \\
\hline Crossing type & -0.104 & 0.000 \\
\hline Number of stops & -0.02 & 0.000 \\
\hline Number of 2W crossings & -0.001 & 0.000 \\
\hline Number of 3W crossings & 0.006 & 0.000 \\
\hline Number of LCVs crossings & -0.002 & 0.000 \\
\hline Number of HCVs crossings & -0.003 & 0.000 \\
\hline Group & -0.007 & 0.000 \\
\hline Crossing time & -0.020 & 0.000 \\
\hline
\end{tabular}

\section{MODEL VALIDATION}

Validation is an essential part of modelling that shows that the model is a realistic representation of an actual system. The Root mean square error (RMSE) and mean absolute percentage error (MAPE) were used for analysing the models validation. MAPE is a useful measure for evaluating a model. RMSE denotes the sample standard deviation, which is calculated by the differences between the predicted values and observed values. The observed and estimated speed values using multiple linear regression (MLR) are plotted in Figure 4. The MAPE values were less than 5\%, and the RMSE values were less than $0 \cdot 5$, meaning that the best-fitting model was validated using actual data.

$$
\text { MAPE }=\frac{\sum_{\mathrm{i}=\mathbf{1}}^{\mathrm{n}}\left|\mathrm{A}_{\mathrm{t}}-\mathrm{F}_{\mathrm{t}}\right|}{\mathrm{A}_{\mathrm{t}}} \times \frac{100 \%}{\mathrm{n}}
$$

where $A_{t}=$ the observed value, $F_{t}=$ the predicted value.

$$
\mathrm{RMSE}=\sqrt{\frac{\sum_{\mathrm{i=1} \mathbf{1}}^{\mathrm{n}}\left(\mathrm{E}_{\mathrm{i}}-\mathrm{O}_{\mathrm{i}}\right)^{2}}{\mathrm{n}}}
$$

$\mathrm{W}$

here $\mathrm{E}_{\mathrm{i}}=$ the expected value, $\mathrm{O}_{\mathrm{i}}=$ the observed value.

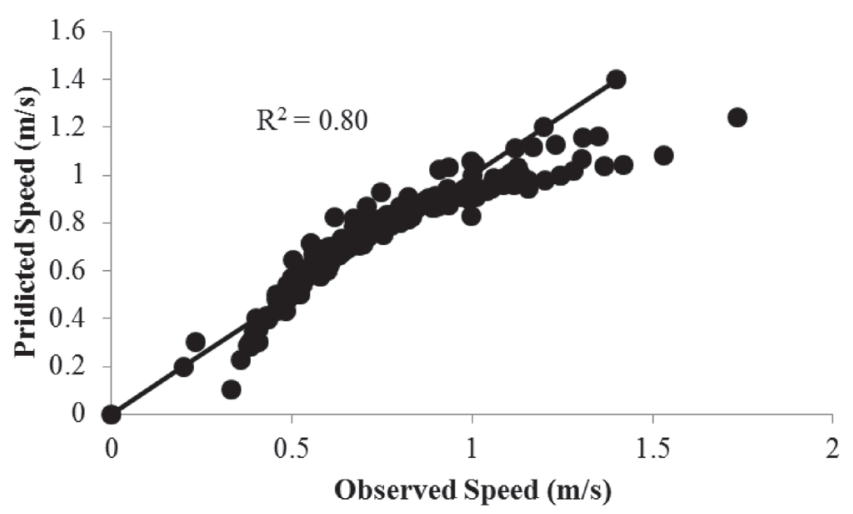

Fig. 4 Observed and predicted crossing speed

\section{CONCLUSIONS}

An experimental study was carried out in order to study the crossing behaviour of pedestrians at a midblock section. The parameters considered were: age, gender, luggage, crossing pattern, number of stops, number of different modes of vehicles crossed, mobile usage, crossing behaviour, number of vehicles making u-turns, and group size. The results show that an older pedestrian's average crossing speed was less than $0.6 \mathrm{~m} / \mathrm{s}$, whereas for children and younger pedestrians, crossing speeds were almost equal i.e, $0.75 \mathrm{~m} / \mathrm{s}$. Middle aged pedestrian speeds were $0.76 \mathrm{~m} / \mathrm{s}$. Female pedestrian speeds were $0.76 \mathrm{~m} / \mathrm{s}$, whereas the average crossing speeds of male pedestrians were $0.67 \mathrm{~m} / \mathrm{s}$. The crossing speeds of pedestrians without luggage were $0.72 \mathrm{~m} / \mathrm{s}$, whereas pedestrians with luggage had crossing speeds of $0.64 \mathrm{~m} / \mathrm{s}$. Pedestrians with a rolling pattern crossing speed were $0.72 \mathrm{~m} / \mathrm{s}$, while they were $0.67 \mathrm{~m} / \mathrm{s}$ for straight patterns. Pedestrians with uninterrupted crossing speeds were $0.8 \mathrm{~m} / \mathrm{s}$, but pedestrians with interrupted crossing speeds were $0.7 \mathrm{~m} / \mathrm{s}$. Crossing speeds were reduced the numbers of times they stopped while crossing the road. Pedestrians using a mobile had a crossing speed of $0.65 \mathrm{~m} / \mathrm{s}$, whereas without a mobile, the speed was $0.72 \mathrm{~m} / \mathrm{s}$. Due to the number of vehicles crossing and types of vehicles crossing speeds were reduced. Pedestrians in a group had a crossing speed of 0.67 $\mathrm{m} / \mathrm{s}$, whereas a pedestrian not in a group had a crossing speed of $0.75 \mathrm{~m} / \mathrm{s}$. Statistical tests were conducted such as ANOVA and 
Pearson correlation tests. The statistical test showed that except for luggage, patterns, the number of u-turning vehicles and mobile usage, other factors significantly affected the crossing speeds of pedestrians. A multiple linear regression model was developed and validated using RMSE and MAPE. This study might be helpful in the management of pedestrian flows at midblock sections; it also suggest to installing a pedestrian signal at midblock locations considering these factors.

\section{REFERENCES}

Kadali, B.R. - Vedagiri, P. (2013) Marked versus unmarked pedestrian road crossing behaviour at uncontrolled midblock crosswalk in mixed traffic condition. Proceedings of $92^{\text {nd }}$ Annual Meeting of the Transportation Research Board (Compendium of Papers, CDROM), No. 13-2943.

Chandra, S. - Rajat, R. - Das, V.R. (2013) Descriptive and parametric analysis of pedestrian gap acceptance in mixed traffic conditions. J Civ Eng 18(1):284-293.

Chu, X. - Guttenplan, M. - Baltes, M.R. (2004) Why people cross where they do: The role of street environment. In Transportation Research Record: Journal of the transportation research board, no. 1878 (pp. 3-10), Washington, D.C.

Daaman, W. - Hoogendorn, S.P. (2007) Pedestrian free speed behavior in crossing flows. Traffic and Granular Flow '05, A. Schadschneider, T. Pöschel, R. Kühane, M. Schreckenberg, and D. E. Wolf, eds., Springer, Berlin, 299-304.

Federal Highway Administration (FHWA). (2003) Manual of uniform traffic control devices for streets and highways, Washington, DC.

Fruin, J.J. (1971) Pedestrian planning and design. Metropolitan Association of Urban Designers and Environmental Planners, New York.

Holland, C. - Hill, R. (2007) The effect of age, gender and driver status on pedestrian's intentions to cross the road in risky situations. Accid. Anal. Prev., 39(2), 224-237.

Lam, W. H. K. - Cheung, C.Y. (2000) Pedestrian speed/flow relationships for walking facilities in Hong Kong. J. Transp. Eng., 126(4), 343-349.

Laxman, K. - Rastogi, R. - Chandra, S. (2010) Pedestrian flow characteristics in mixed traffic conditions. Journal of Urban Planning And Development 136 (1), 23-33.

Leather, J. - Fabian, H. - Gota, S. - Mejia, A. (2011) Walkability and Pedestrian Facilities in Asian Cities: State and Issues. ADB Sustainable Development Working Paper Series. Asian Development Bank, Manila,he Philippines, 78 pp.
Mohan, D. - Tsimhoni, O. - Sivak, M. - Flannagan, M. J. (2009) Road safety in India: Challenges and Opportunities. Transport Research Institute, University of Michigan. 62 pp.

Papadimitriou, E. - Yannis, G. - Golias, J. (2009) A critical assessment of pedestrian behavior models. Transportation research part F., 12, 242-255.

Rajat, R. - Chandra, S. - Vamsheedhar, J. - Das, V.R. (2011) Parametric study of pedestrian speeds at midblock crossings. Journal of Urban Planning and Development, 137(4), 381-389.

Shaaban, K. - Muley, D. - EInashar, D. (2017) Evaluating the effect of seasonal variations on walking behaviour in a hot weather country using logistic regression. International Journal of Urban Sciences, 22(3), 382-391. doi:10.1080/12265934.2017.1403363.

Tanaboriboon, Y. - Guyano, J. A. (1991) Analysis of pedestrian movements in Bangkok. Transportation Research Record 1294, Transportation Research Board, Washington, DC, 52-56.

Tarawneh, M.S. (2001) Evaluation of pedestrian speed in Jordan with investigation of some contributing factors. J. Saf. Res., 32(2), 229-236.

Transportation Research Board (TRB). (2000) Highway capacity manual. Special Rep. No. 209, National Research Council, Washington, DC.

Yannis, G. - Papadimitriou, E. - Theofilatos, A. (2013) Pedestrian gap acceptance for mid block street crossing. Transportation Planning and Technology, 36(5), 450-462.

Zhao, J. - Malenje, J. O. - Tang, Y. - Han, Y. (2019) Gap acceptance probability model for pedestrians at unsignalized midblock crosswalks based on logistic regression. Accident Analysis \& Prevention, 129, 76-83. doi:10.1016/j.aap.2019.05.012.

Zhuang, X. - Wu, C. (2011) Pedestrians' crossing behaviors and safety at unmarked roadway in China. Accident Analysis \& Prevention, 43, pp. 1927-1936. 